Edinburgh Anisotropy Project

12iwsa-xyl.doc

Feb 2007

\title{
Estimating anisotropic parameters from PS converted-wave data: a case study
}

Xiang-Yang Li $i^{l}$,Lifeng Wang $^{1}$, Hengchang Dai ${ }^{1}$ and Xianyi Sun ${ }^{2}$

\footnotetext{
${ }^{1}$ British Geological Survey, West Mains Road, Edinburgh EH9 3LA, UK

${ }^{2}$ PetroChina Daqing Ltd. Helongjiang, China

Paper presented at the $12^{\text {th }}$ International Workshop on Seismic Anisotropy - Beijing, 22-25 $5^{\text {th }}$ October 2006
}

Suggested short title: Estimating anisotropy from converted-wave data

Intended publication: Journal of Applied Geophysics

Submitted on

27 February 2007

Revised on

*Address for correspondence

Xiang-Yang Li

Britigh Geological Survey

Murchison House,

West Mains Road

Edinburgh EH9 3LA

Scotland,UK

xyl@bgs.ac.uk 


\begin{abstract}
Anisotropic parameters are estimated from PS converted-wave ( $C$-wave) data. The data were acquired by digital MEMS (micro-electro-mechanical system) sensors over a volcanic gas reservoir in Northeast China with a mixed sand and shale sequence in the overburden. This gives rise to both shear-wave splitting and anisotropy moveout effects due to presence of vertical transverse isotropy (VTI). We use a four-parameter theory to evaluate the effects of non-hyperbolic moveout due to an asymmetric raypath and VTI. These four parameters include the PS converted wave stacking velocity $\left(V_{\mathrm{C} 2}\right)$, the vertical velocity ratio $\left(\gamma_{0}\right)$, the effective velocity ratio $\left(\gamma_{\text {eff }}\right)$, and the anisotropy parameter $\left(\chi_{\text {eff }}\right)$. This four-parameter theory leads to an improvement in imaging quality and correlation between the P-waves and converted-waves. After compensating for the VTI effects, we analyze the shear-wave splitting in the post stacked data. We find that the amount of splitting determined from the data can be correlated to the known gas reservoirs, and is also consistent with other amplitude and spectral anomalies associated with the gas reservoirs, revealing a potential for using shear-wave splitting to delineate gas reservoirs in volcanic rocks.
\end{abstract}

Keywords: Converted-wave, shear-wave splitting, transverse isotropy, VTI

\title{
1. Introduction
}

It has become increasingly common knowledge that anisotropy is widely present in the Earth subsurface. Sedimentary layers such as shales and thin bedding sequences in the overburden often give rise to vertical transverse isotropy (VTI, or polar anisotropy), whilst the presence of near-vertical fractures in sand or carbonate hydrocarbon reservoirs may result in horizontal transverse isotropy (HTI, or azimuthal anisotropy). For VTI, a major seismic effect is non-hyperbolic moveout, whilst for HTI, we have shear-wave splitting, both of which are intensively studied in the literature (e.g. Helbig and Thomsen, 2005, Crampin and Lovell, 1991). However, due to data quality issues, it is not very common to find both of these effects present in a land shear-wave dataset that can be clearly identified and used for reservoir characterization.

Recently, the use of digital MEMS (micro-electro-mechanical system) sensors has substantially improved the quality of land converted-wave data (e.g. Calvert et al., 2005). As a result, here we present an example of using MEMS-based converted-wave technology for jointly evaluating the effects of VTI and shear-wave splitting in Northeast China. The data 
were recorded over a volcanic gas reservoir buried at depths ranging from $2800 \mathrm{~m}$ to $3600 \mathrm{~m}$, and the overburden is primarily composed of a mixed sand and shale sequence. The experiment acquired a total of six 2D survey lines (Chen et al., 2007), and we will analyze one of the lines (Line 2) in this study.

The use of digital MEMS sensors has lead to the acquisition of very high quality shearwave data (Fig. 1), and major events can be clearly identified in the original shot record, although some ground roll can be observed. Dominant frequency is about $40 \mathrm{hz}$ for P-wave and about $20 \mathrm{hz}$ for converted-wave. There is a significant amount of energy present in the crossline (transverse) component, indicating the presence of shear-wave splitting (Fig. 1b). Whilst in the shallow overburden, significant non-hyperbolic moveout can also be observed, which cannot be corrected fully by accounting for the asymmetric raypath. This paper studies the effects of non-hyperbolic moveout on imaging quality and event correlation. The paper also evaluates the effects of shear-wave splitting and its application for characterizing gas reservoirs.

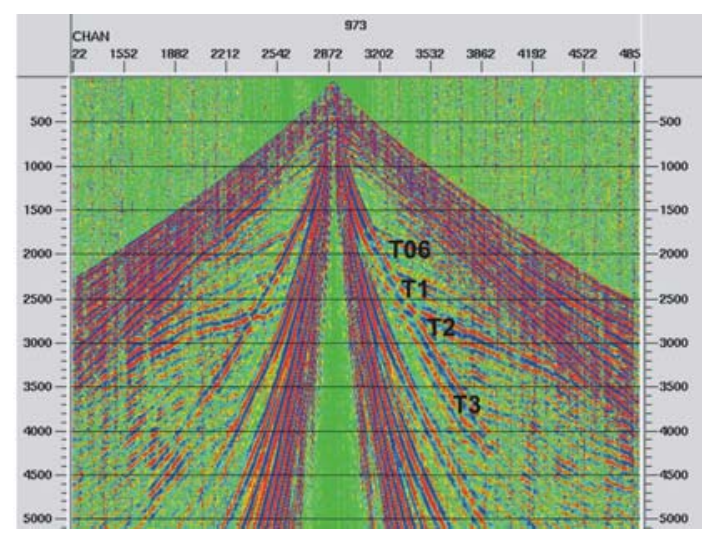

(a) Inline (radial, $x$ )

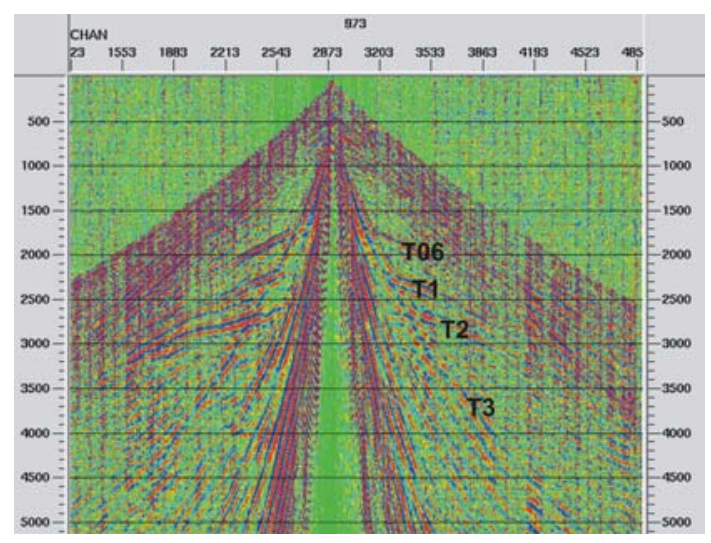

(b) Crossline (transverse, y)

Fig. 1. Sample shot gathers of PS Converted-wave: (a) inline (Radial) and (b) crossline (transverse) components. Symbols T06, T1, T2 and T3 mark the main horizons in the study area.

\section{Analysis of VTI effects}

Due to the improved data quality, the workflow to perform anisotropic processing is relatively straightforward. The following nine steps are applied to both the vertical (PP-) and horizontal (PS-) components: 1) geometry loading; 2) static correction; 3) noise attenuation (ground roll); 4) CMP bin for PP, but ACP (Asymptotic conversion point) bin for PS with binning velocity ratio $\gamma=2.0$; 5) velocity analysis, 6) moveout correction; 7) stack; 8) migration velocity analysis 
and 9) final prestack time migration. In the following sections, we will discuss some data preprocessing issues and present the processing results in the presence of VTI.

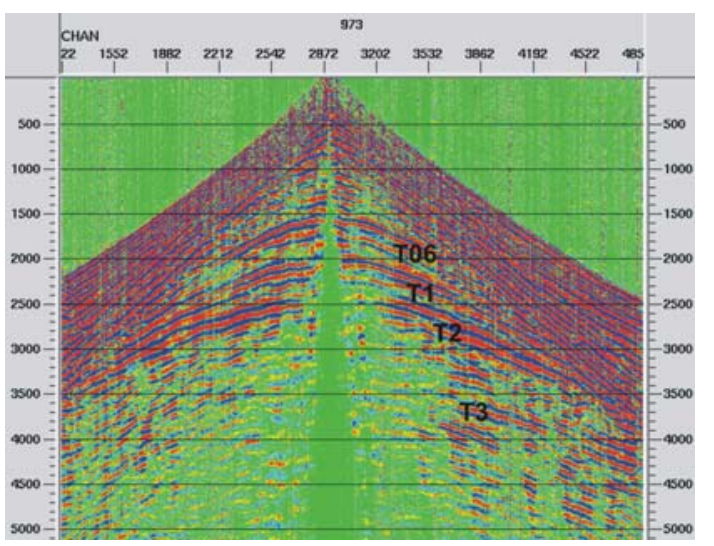

(a) Inline (radial, $x$ )

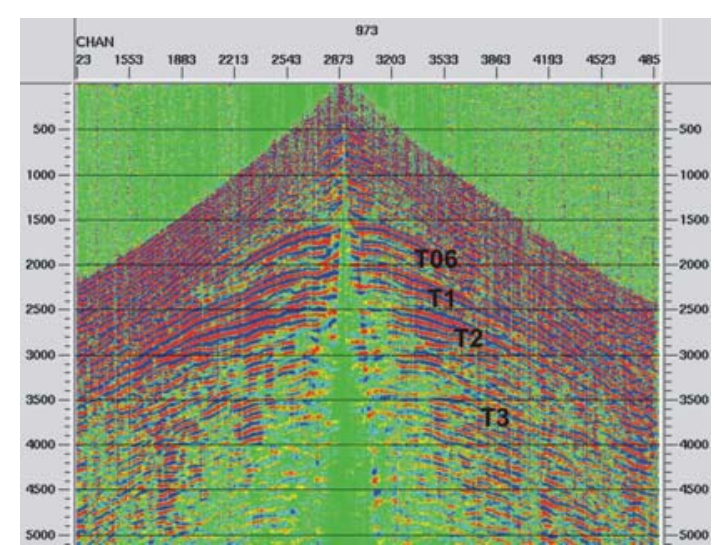

(b) Crossline (transverse, y)

Fig. 2. The same as Fig. 1, but after applying noise reduction using a model-based technique (Qian and Zhao, 2003).

\subsection{Data processing and noise reduction}

Statics and noise are two common problems for land converted-wave data. Fortunately, in this case, the study area is relatively flat and we do not have a serious static issue. Elevation static correction combined with a residual static correction is sufficient for this dataset. In contrast, noise reduction for improving signal-to-noise ratio is more urgent. In this dataset, the main problem in noise reduction is how to remove the ground roll that is widely present in the data. In conventional data acquisition, array forming is usually used to reduce the ground roll. Here, data are acquired using single-point sensors, and severe ground roll can be observed in the data, as shown in Fig. 1. Due to the strong presence of the ground roll, conventional F-K filtering techniques are often inadequate. For this dataset, we developed a deterministic model-based technique (Qian and Zhao, 2003), and applied to the data. The ground roll is effectively removed, as shown in Fig. 2.

\subsection{Estimating anisotropic parameters for VTI}

Note that in the processing of the converted-wave, in addition to the noise problem, another problem is the non-hyperbolic moveout due to the asymmetry of raypath and anisotropy, in particular, vertical transverse isotropy (VTI). According to Li and Yuan (2003), four parameters are required to perform converted-wave velocity analysis and moveout 
correction in the presence of VTI. These four parameters are the vertical $P$ - and $S$-wave velocity ratio $\gamma_{0}$, the effective velocity ratio $\gamma_{\text {eff }}$, the converted-wave stacking velocity $V_{\mathrm{C} 2}$ and anisotropic parameter $\chi_{\text {eff. }}$ The PS converted-wave (C-wave) moveout equation in horizontally layered VTI media can then be expressed as,

$t_{C}^{2}=t_{C 0}^{2}+\frac{x^{2}}{V_{C 2}^{2}}+\frac{A_{4} x^{4}}{1+A_{5} x^{2}}$,

where

$A_{4}=-\frac{\left(\gamma_{0} \gamma_{e f f}-1\right)^{2}+8\left(1+\gamma_{0}\right) \chi_{e f f}}{4 t_{C 0}^{2} V_{C 2}^{4} \gamma_{0}\left(1+\gamma_{e f f}\right)^{2}}$, and $A_{5}=\frac{A_{4} V_{C 2}^{2}\left(1+\gamma_{0}\right) \gamma_{e f f}\left[\left(\gamma_{0}-1\right) \gamma_{e f f}^{2}+2 \chi_{e f f}\right]}{\left(\gamma_{0}-1\right) \gamma_{e f f}^{2}\left(1-\gamma_{0} \gamma_{e f f}\right)-2\left(1+\gamma_{0}\right) \gamma_{e f f} \chi_{e f f}}$.

Eqs. (1) and (2) are accurate for offset-depth ratio up to $2.0(x / z \leq 2.0)$ (Li and Yuan, 2003). Eq. (1) controls the stacking process, and these four parameters are referred to as the C-wave stacking velocity model. Note that $\gamma_{\text {eff }}$ is related to $\gamma_{0}$ and the $P$ - and $S$-wave NMO stacking velocity ratio $\gamma_{2}\left(\gamma_{\text {eff }}=\gamma_{2}^{2} / \gamma_{0}\right) \cdot \chi_{\text {eff }}$ measures the amount of converted-wave traveltime anisotropy, and is a combination of the P-wave and S-wave anisotropy parameters (See Li and Yuan 2003 for more details).

The following work flow may be used to determine these four parameters $\left(V_{\mathrm{C} 2}, \gamma_{0}, \gamma_{\mathrm{eff}}\right.$ and $\left.\chi_{\text {eff }}\right)$ : 1 ) obtain $\gamma_{0}$ by a coarse correlation between the $P$ - and $C$-wave stacked sections; 2) estimate $V_{\mathrm{C} 2}$ from the near-offset moveout signature (offset-depth ratio $x / z<1.0$ ); 3) estimate $\gamma_{\text {eff }}$ from the intermediate-offset moveout $(x / z<1.5)$ and $\chi_{\text {eff }}$ from the far offset moveout $(x / z<2.0)$. An interactive velocity tool has been developed by Dai (2003), which can be used to perform the above steps.

\subsection{Processing results for VTI}

Examples of anisotropic velocity analysis of the radial component are shown in Fig. 3. In the shallow section, there is still significant residual moveout without considering anisotropy (Fig. 3a - setting $\chi_{\text {eff }}$ to zero), whereas the event is properly aligned after taking account for anisotropy (Fig. $3 \mathrm{~b}-$ non-zero $\chi_{\text {eff }}$ ). We can see that the moveout correction is very sensitive to the presence of VTI. Using the interactive tool as shown in Fig. 3, we can determine values of $V_{\mathrm{C} 2}, \gamma_{\mathrm{eff}}$ and $\chi_{\mathrm{eff}}$ across the whole section, as shown in Fig.4. We can see that the amount of anisotropy is very significant for the events around 2.0 seconds (Fig. 4d). In comparison, the other quantities ( $V_{\mathrm{C} 2}, \gamma_{0}$ and $\gamma_{\text {eff }}$ ) all vary smoothly. 
(a)

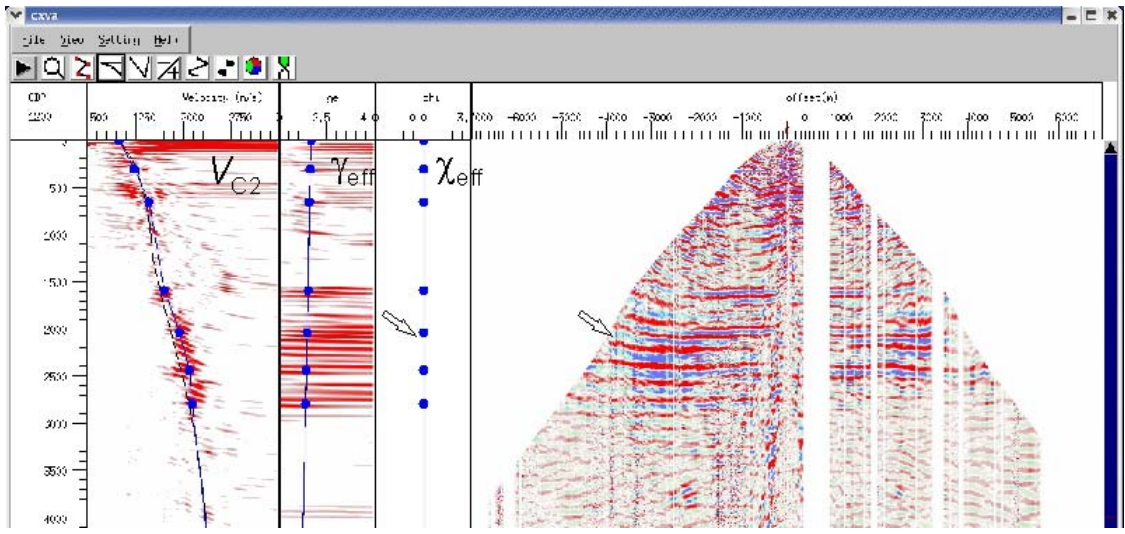

(b)

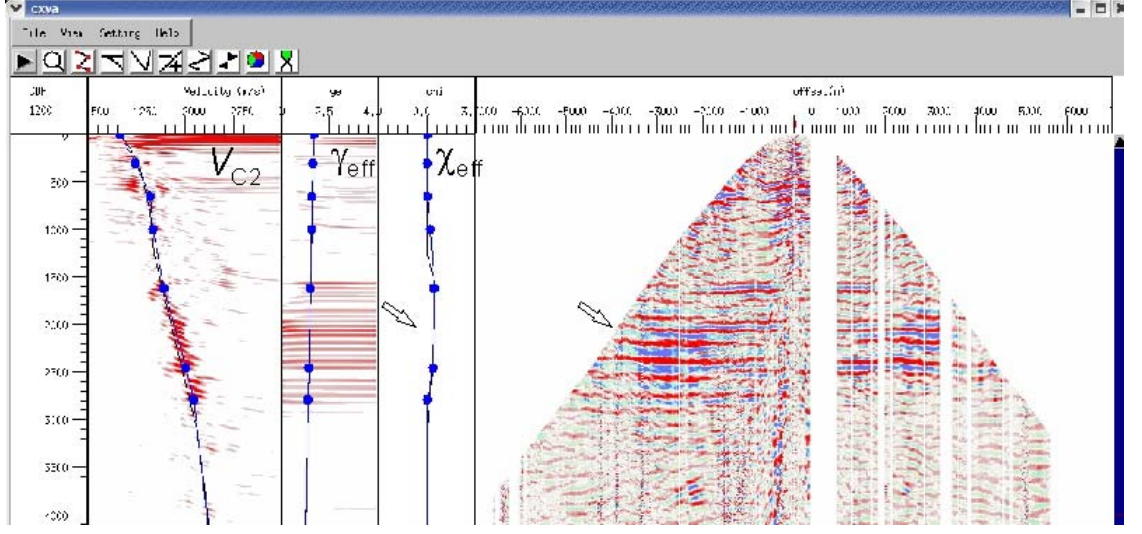

Fig. 3. Analysis of the effects of anisotropy on converted-waves: velocity analysis and moveout correction (a) without and (b) with anisotropy. Note that from left to right, the three panels show the semblance analyses for $\mathrm{V}_{\mathrm{C} 2}, \gamma_{\mathrm{eff}}$ and $\chi_{\text {eff }}$, respectively. The data are from the inline radial component.

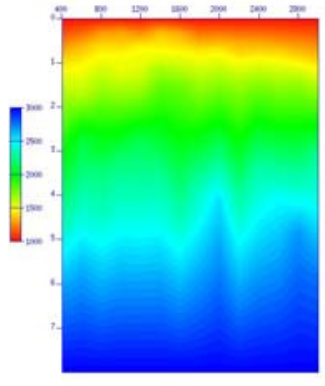

(a) $V_{\mathrm{C} 2}$

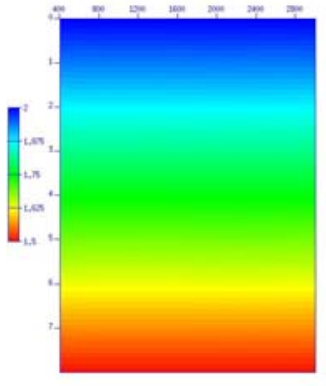

(b) $\gamma_{0}$

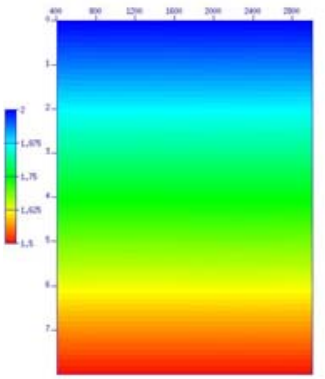

(c) $\gamma_{\text {eff }}$

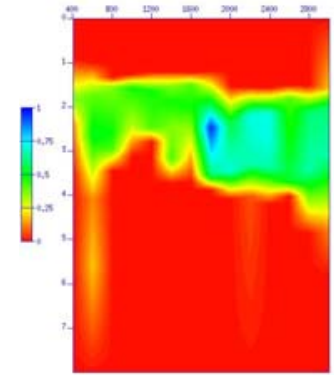

(d) $\chi_{\text {eff }}$

Fig. 4. The four parameters (a) $V_{\mathrm{C} 2}$, (b) $\gamma_{0}$, (c) $\gamma_{\text {eff }}$ and (d) $\chi_{\text {eff }}$ estimated from C-wave moveout analysis using the interactive tools as shown in Fig. 3 for the radial-component . 
All the data are processed using the above flows. The stacked velocity model derived from Fig. 4. is used as an initial model for prestack time migration in order to generate common imaging gathers ( $\mathrm{Li}$ et al., 2007). These gathers are then used to update the velocity model for final migration. The final migrated PP- and PSV-sections are shown in Fig. 5, where the regional events (T06, T1 and T2, etc.) can all be mapped from both the PP- and PSsections, giving rise to a very high degree of correlation. The faulting in the shallow part of section is also well imaged.

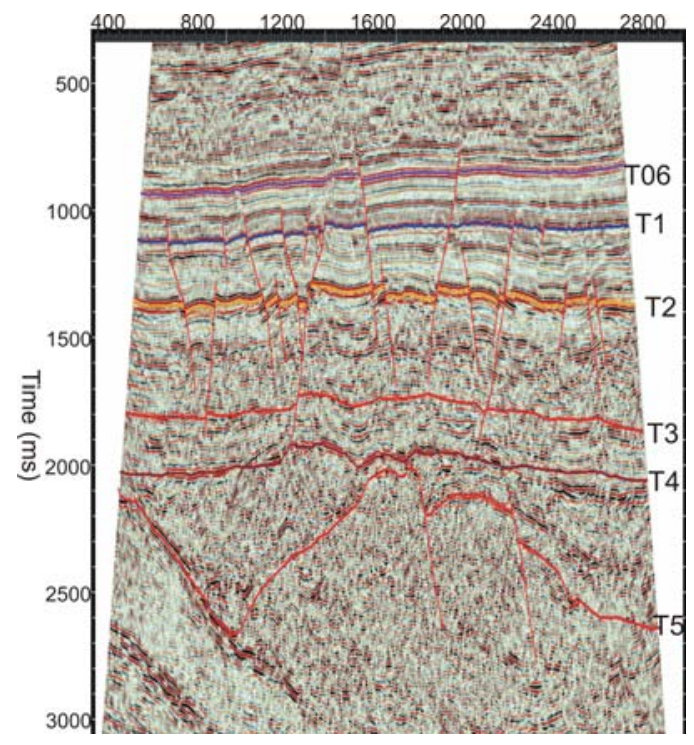

(a) PP-section

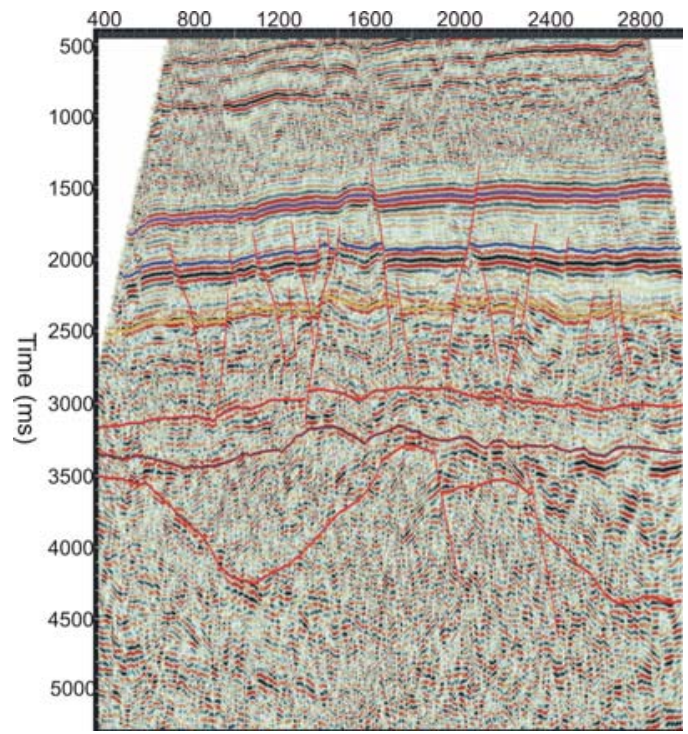

(b) PS-section (Radial component)

Fig. 5. Comparison of final migrated sections: (a) PP-wave and (b) PSV converted wave (Radial component).

\section{Shear-wave splitting analysis}

When a PS conversion occurs at a reflector and the medium above the reflector is azimuthally anisotropic, the converted shear wave splits into a fast and a slow shear wave, and this is referred to as converted-wave splitting ( $\mathrm{Li}, 1998)$. The splitting can be caused by the alignment of cracks, fractures, and small heterogeneities in the reservoirs. There is a wide interest from the industry to use converted-wave splitting to evaluate the internal architecture of the reservoirs. In particularly, there is a vast amount of literatures on the use of shear-wave or converted-wave splitting to characterize fractured reservoirs (e.g. Li, 1997; Gaiser and Van Dok 2001; Angerer et al, 2002; Vetri et al. 2003; amongst others)

Shear-wave splitting in volcanic rocks has been reported before (e.g. Crampin and Lovell 1991). Volcanic rocks are known to be heterogeneous and can contain a large amount 
of fractures and vuggy pores, as well as other small heterogeneities. When these small-scale features are gas-charged and stress-aligned, it will introduce azimuthal anisotropy, giving rise to shear-wave splitting. In the previous sections, we have analyzed the vertical component (PPwave) and the horizontal radial component (PSV-waves) for VTI. Here we focus only on the two horizontal components for shear-wave splitting.

\subsection{Basic analysis methods}

Most of the rotation algorithms for shear-wave splitting, such as Alford rotation (1986) and the linear transform technique ( $\mathrm{Li}$ and Crampin 1993), require multi-azimuth distribution. However, the converted-wave data used in this study are acquired with a 2D configuration and single-azimuth source and receiver distribution. For single-azimuth data, a rotation scanning procedure is often needed that searches for optimum solutions according to certain criteria. One of the common criteria is the similarity of waveforms between the fast and slower shearwaves. Here we adopt a criterion that maximizes the separation of the fast and slow shearwaves, following the approach of Yuan (2001).

Fig. 6 shows a map view of shear wave splitting when an up-going converted shear wave travels in a medium with a preferred distribution of heterogeneities. The principal direction of medium forms an oblique angle $\theta$ with the survey line. The orientation of the three component geophone forms a right-handed coordinate system: Radial component $\mathrm{R}$ is the inline horizontal, transverse component $\mathrm{T}$ crossline horizontal and vertical component $\mathrm{V}$, point to the reader. The polarization of the fast split shear wave $S_{1}$ is parallel to the principal direction and that of the slow shear wave $S_{2}$

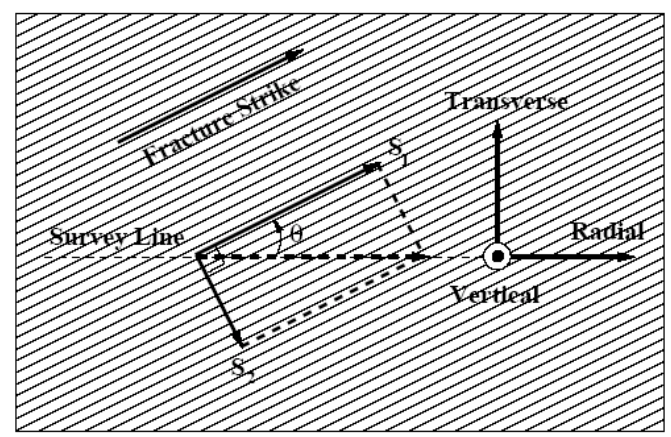

Fig. 6. The coordinate system used for shear-wave splitting analysis. perpendicular to it.

To process the split shear waves, it is common to use the convolution model of seismic wave propagation. Thus, in the frequency domain, the wavefield recorded by the radial and transverse components can be written as,

$$
\left[\begin{array}{c}
R(\omega) \\
T(\omega)
\end{array}\right]=\left[\begin{array}{cc}
\cos \theta & \sin \theta \\
\sin \theta & -\cos \theta
\end{array}\right]\left[\begin{array}{l}
S_{1}(\omega) \\
S_{2}(\omega)
\end{array}\right]+\left[\begin{array}{c}
N_{R}(\omega) \\
N_{T}(\omega)
\end{array}\right]
$$


where $N_{R}(\omega)$ and $N_{T}(\omega)$ are the noise components in the radial and transverse directions. To maximize the separation between the fast and slow shear-wave, one may calculate the residual errors between the fast and slow waves as

$$
E(\theta, \Delta t, \omega)=S_{1}(\omega)-S_{2}(\omega) e^{i \omega \Delta t}
$$

where $\Delta t$ is time delay between the fast and slow waves. Substituting into eq. (1) and changing into the time domain, it becomes,

$$
\begin{aligned}
& E(\theta, \Delta t, t)=(R(t) \cos \theta+T(t) \sin \theta)-(R(t-\Delta t) \sin \theta-T(t-\Delta t) \cos \theta) \\
& -\left(N_{R}(t) \cos \theta+N_{T}(t) \sin \theta\right)+\left(N_{R}(t-\Delta t) \sin \theta-N_{T}(t-\Delta t) \cos \theta\right)
\end{aligned}
$$

Eq. (5) forms the basis for our analysis. One can perform double scanning over the rotation angle $\theta$ and time delay $\Delta t$. For this we define an objective function $F(\theta, \Delta t)$ in order to minimize the summed $E(\theta, \Delta t, t)$ as

$$
F(\theta, \Delta t)=\left(\sum_{k=0}^{n} E\left(\theta, \Delta t, t_{k}\right)^{p}\right)^{1 / p}
$$

over a time window length with n samples.

\subsection{Time delay spectra}

Once the split shear-waves are separated, we construct a time-delay spectrum between the fast and slow waves that allows the picking of time delays as a function of the vertical travel time, yielding a time-delay section for interpretation purposes. The time-delay spectrum can be constructed by scanning over vertical time using the correlation method. For each trace pair of the fast and slow split shear-waves, we use a sliding time-window to compute the correlation coefficients within the window, which produces a time-delay spectrum.

\subsection{Results of splitting analysis}

In order to maintain the characteristics of shear-wave splitting, the two horizontal components are processed using the same processing flow as described previously, and the analysis is performed on the un-migrated stacked data (Fig. 7). Strong coherent energy can be observed in the transverse component, indicating shear-wave splitting (Fig. 7b). Note that in the absence of splitting, the transverse component should contain mainly noise. The rotationscanning method and time-delay spectrum analysis described previous are applied to the data. 


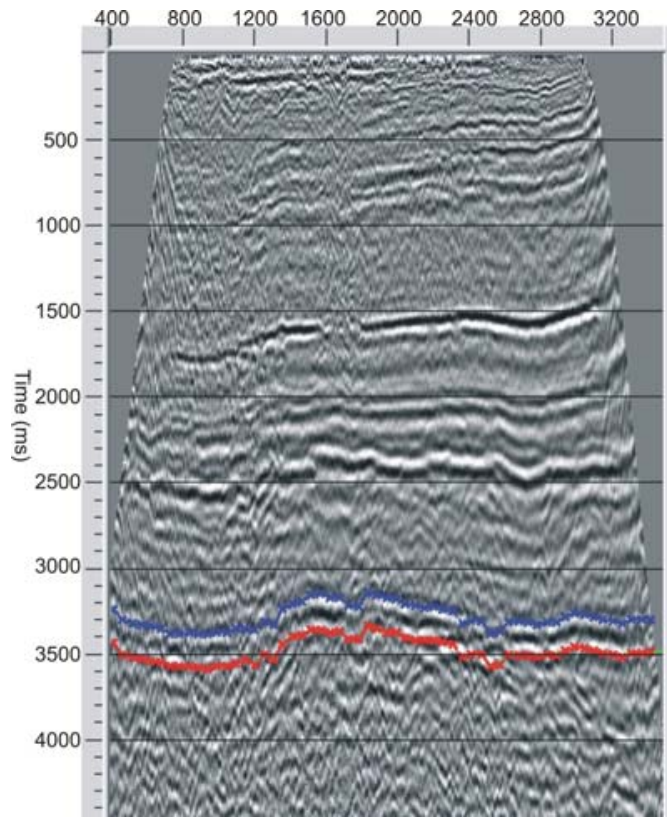

(a) Radial

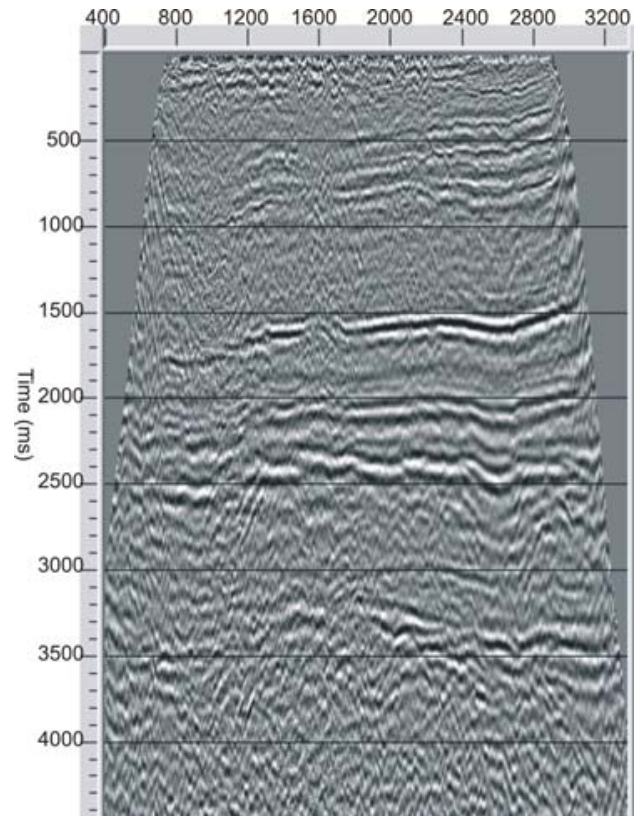

(b) Transverse

Fig. 7. Input data for shear-wave splitting analysis: (a) radial and (b) transverse components. The blue and red lines show the top and bottom of the reservoir target.

Fig. 8 shows contour displays of the objective function of eq. (6) for some selected CDPs over the target horizon marked by the blue and red lines in Fig. 7. The purpose of rotation scanning is to minimize the objective function, and the corresponding rotation angle and timedelay at the minimum point of the objective function are the desired solutions. This analysis is repeated for every CDPs, and findings are displayed in Fig. 9. We can see that the average orientation angle is about 40 degrees from the inline direction (anticlockwise) and the average time delay is $25-30$ milliseconds, indicating about $2 \%$ shear-wave splitting (Fig. 9). The data are then rotated into the fast and slow components for calculating the time-delay spectras. Fig. 10 shows the time-delay spectra from selected CDP locations, from which a time-delay section can be obtained. Fig. 11 is the interpretation results on the time-delay gradient section. As shown in Fig. 11, areas of volcanic rocks with high gas accumulation show a significant amount of splitting (wells SS202 and SS1), whilst non-gas bearing rocks show little splitting (well SS201). The splitting anomalies seem to be good indicators of gas accumulation. 


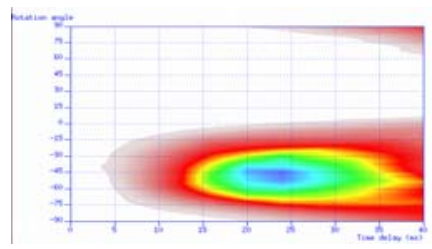

CDP 400

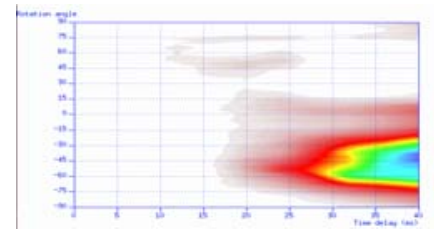

CDP 1000

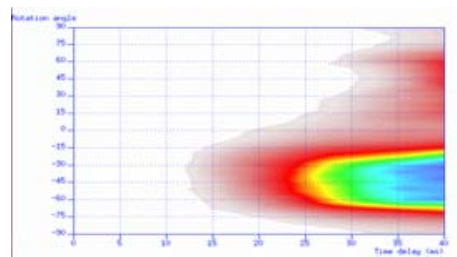

CDP 600

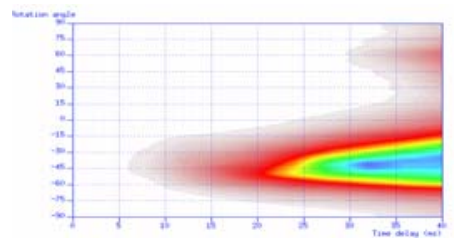

CDP 1200

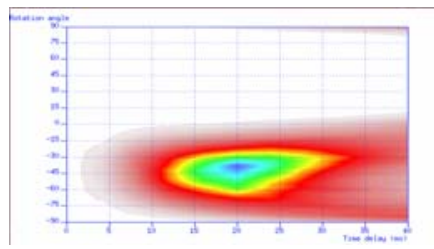

CDP 800

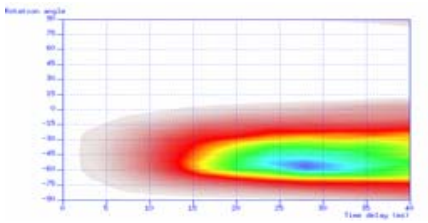

CDP 1400

Fig. 8. The scanning is looking for a minimum value (in blue). The vertical axis is rotation angle, and the horizontal axis is time-delay.

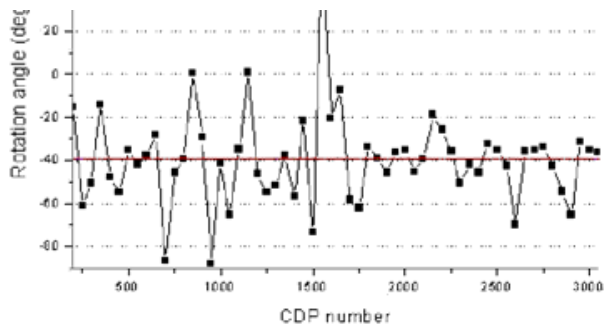

(a) Polarization angle

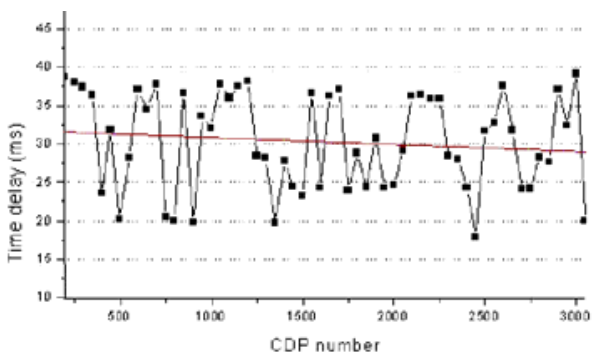

(b) Time delays

Fig. 9. Picked (a) polarization angles of the fast split shear-wave and (b) time delays of the split shear-waves from the scanning results in Fig. 8. 


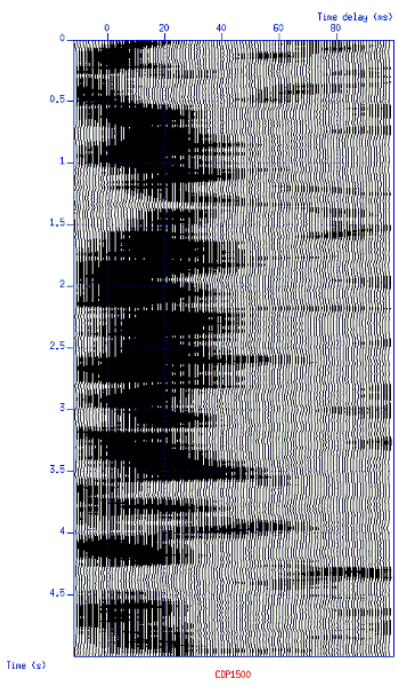

CDP 1500

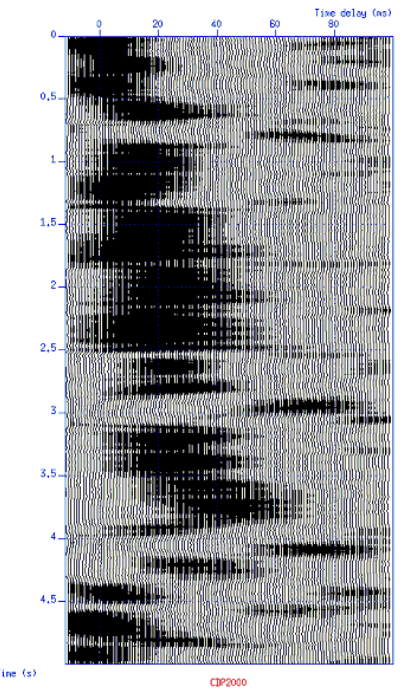

CDP 2000

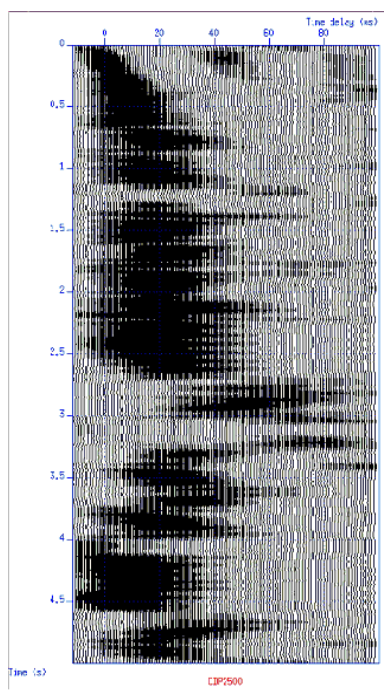

CDP 2500

Fig. 10. Time-delay spectra of selected CDPs. The horizontal axis is time-delay, and the vertical axis is two-way travel time.

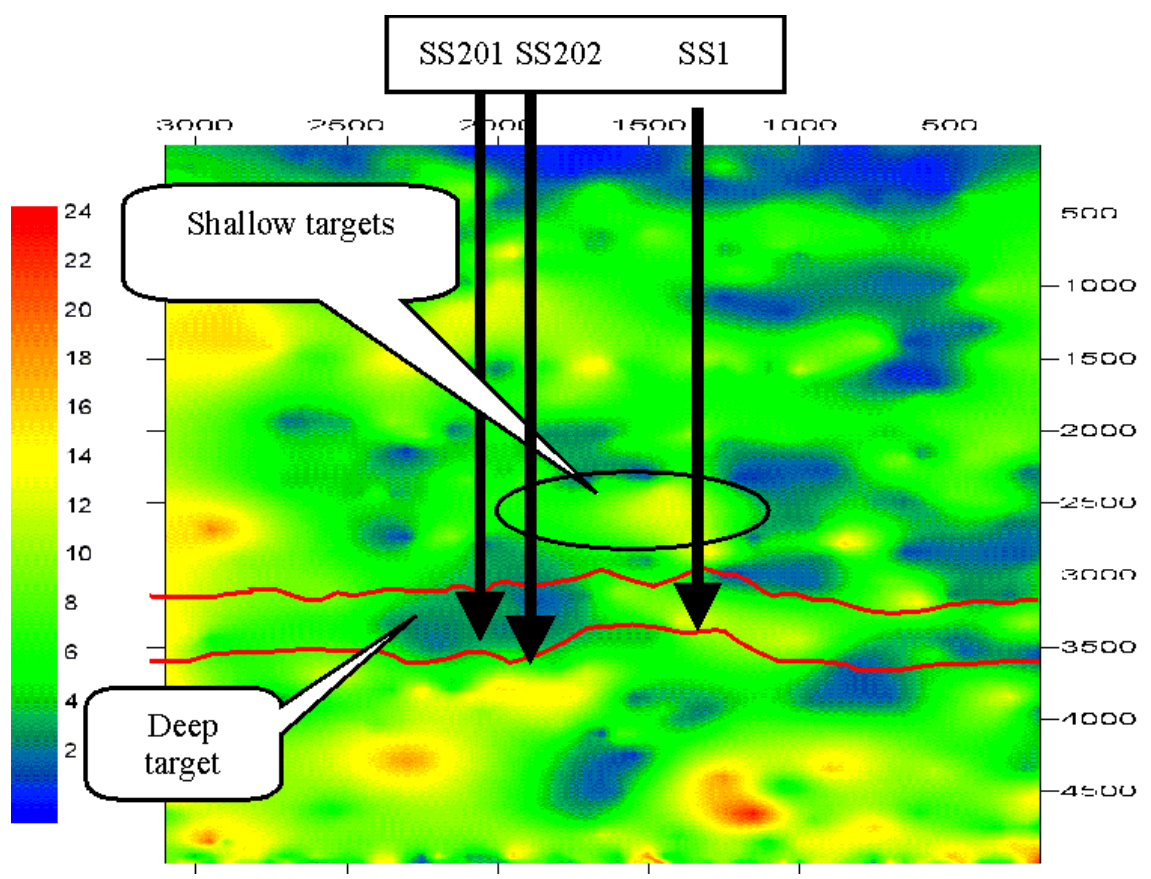

Fig. 11. Interpretation results on the time-delay gradient section. SS202 and SS1 are gas-producing wells, and SS201 is a dry hole. 


\section{Analysis of amplitude characteristics}

We have shown the amount of shear-wave splitting increasing with gas accumulation. Here, we analyse the other wavefield characteristics associated with the volcanic gas reservoirs. These include P- and S-wave amplitudes and their spectral characteristics, all of which show consistent diagnostics of the gas reservoir.

\subsection{Variations in the PP- and PS-wave amplitudes}

The presence of gas in the volcanic rock reduces P-wave velocity, whilst its effects on the Swaves are relatively small (Jiang et al., 2004), since the S-wave is generally less sensitive to the presence of fluids than the P-wave. This leads to clear anomalies in the P- and S-wave velocity ratio ( $\mathrm{Vp} / \mathrm{Vs}$ ratio). $\mathrm{Vp} / \mathrm{Vs}$ ratio anomalies have also been observed in this area (Chen et al. 2007), here we focus on the amplitude characteristics. The presence of gas in the volcanic rock also increases the attenuation and scattering effects on the P-wave, whilst the S-wave remains almost unaffected. This in turn leads to distinct anomalies in the P- and S-wave amplitudes.

Fig. 12 compares the PP- and PS-amplitude from the gas producing well SS202, and the dry hole SS201. The P-wave reflection from the gas formation is consistently weak, and the events are more scattered, as indicated by the red box and the red and yellow arrows. In contrast, the PS-reflection is much stronger and the events are more continuous. In comparison, for the non-producing well, both PP- and PS-reflection are very strong and continuous, as shown in Fig. 12b.

\subsection{Variation of reflection amplitude with frequency}

Castagna et al. (2003) showed that the presence of a low-frequency shadow in the P-wave seismic section is often a good indicator of gas accumulation, and they then used spectral decomposition to analyse this phenomenon. This can be modelled using a multi-scale rock physics model proposed by Chapman (2003), as demonstrated in Odebeatu et al. (2006). The Chapman model (2003) also predicts that compared with the P-wave, there is less attenuation of the PS-wave. That is, whilst there may exist low-frequency gas shadows in the P-wave section, the PS-wave may not show such obvious changes of energy with frequency. Here, we analyse this dataset and evaluate the variations of PP- and PS-reflection energy with frequency. 


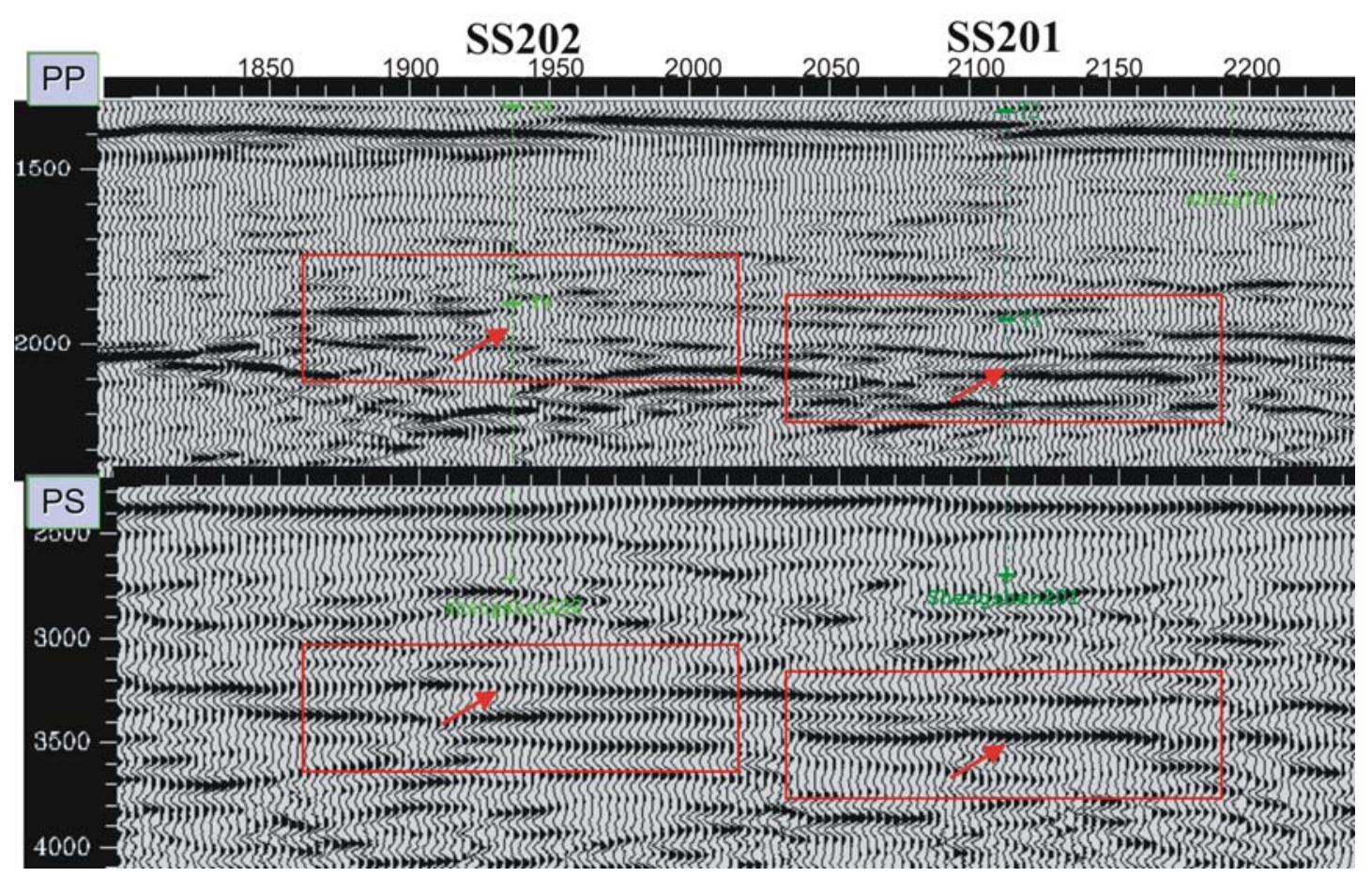

Fig. 12. Comparison of PP- and PS amplitude characteristics for the volcanic formation: from the gas producing well SS202, and the dry hole SS201.

Using spectral decomposition, we can examine the variation of reflection energy with frequency following Castagna et al. (2003). Figs. 13 and 14 show the reflection energy of PP and PS -waves at different frequency bands. We select five bands: 10hz, 15hz, 20hz, 25hz and $30 \mathrm{hz}$ for the PP-wave; $5 \mathrm{hz}, 8 \mathrm{hz}, 10 \mathrm{hz}, 13 \mathrm{hz}$ and $16 \mathrm{hz}$ for the PS-wave. All the energy spectra are normalized against a reference horizon at 1.3s P-wave time (Fig. 13), and 2.5s PS-wave time (Fig. 14). The ellipses identify the volcanic targets. In the P-wave section (Fig. 13), bright spots for the gas-producing wells SS1 and SS202 can be identified in the panels at 10hz and 15 hz. These bright spots disappear gradually, as frequency increases. In contrast, the reflection energy for the dry well SS201 remains almost constant across the frequency bands. Furthermore, the reflection energy in the PS section also shows no obvious various with the frequency bands for all three wells. This agrees with the theory, and consistent with shearwave splitting anomalies in Fig. 11 and the amplitude anomalies in Fig. 12. 

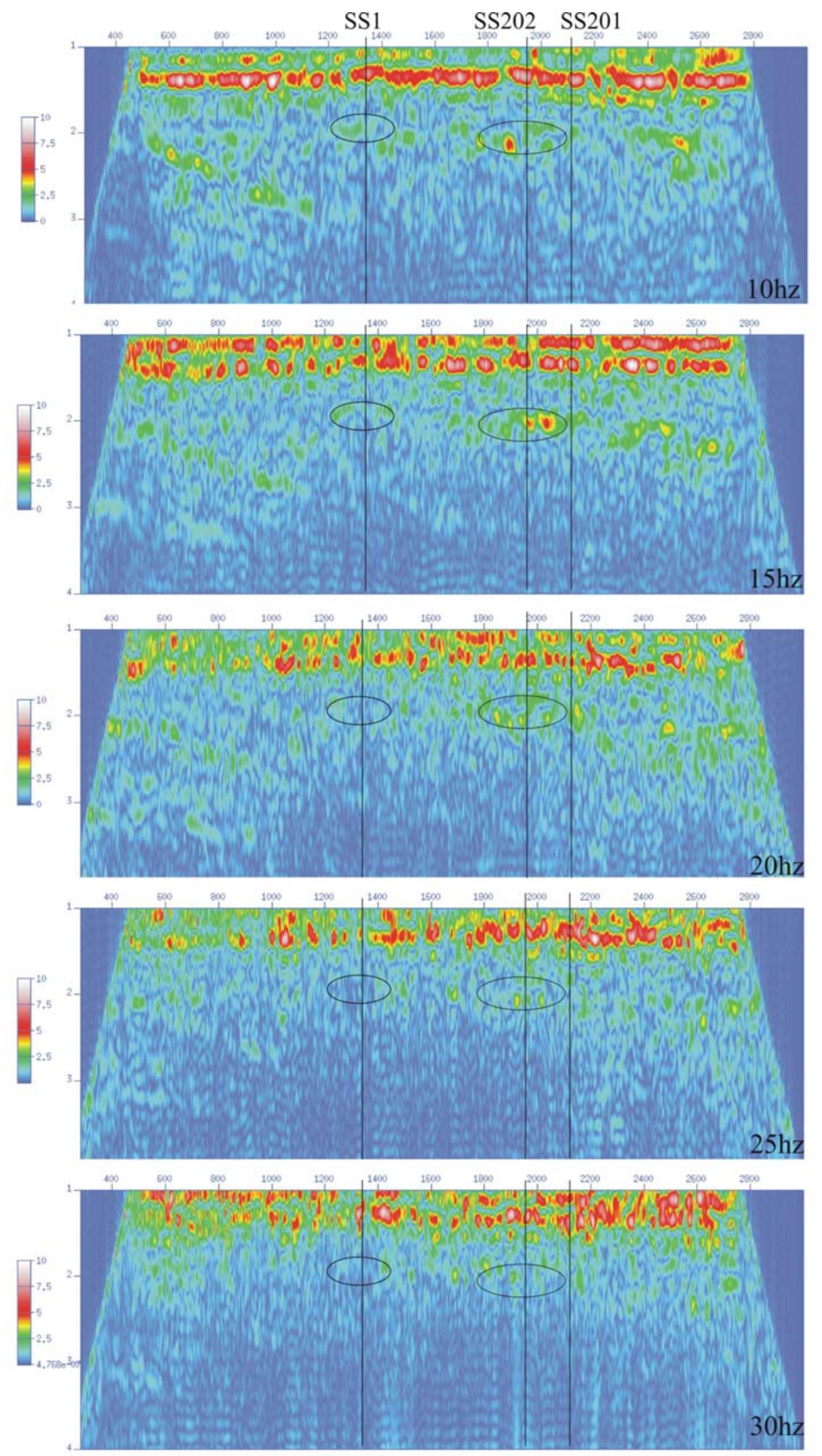

Fig. 13. Variation of reflection energy with frequency in the PP section. Wells SS1 and SS202 are gas-producing wells and SS201 is a dry hole. 

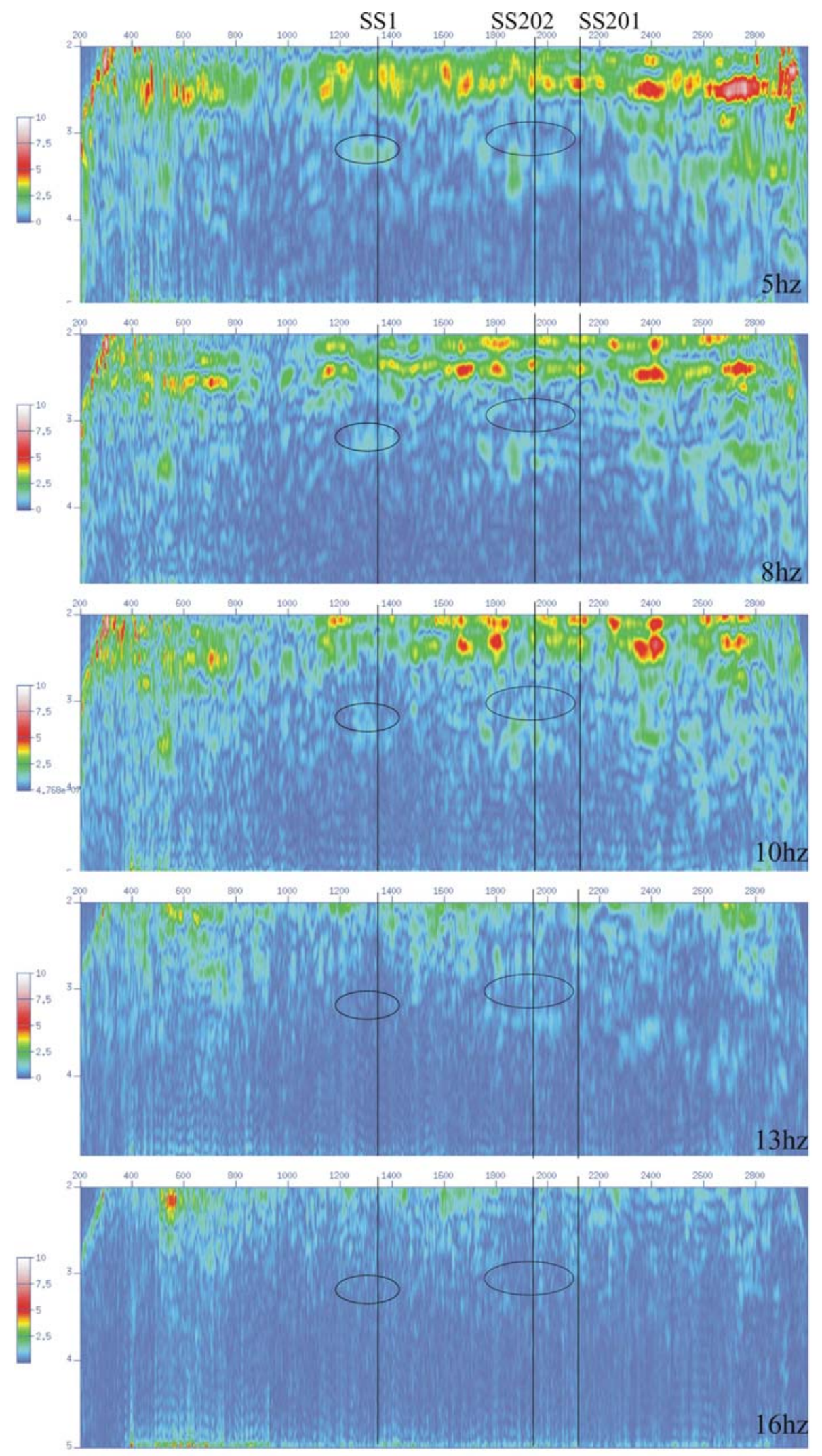

Fig. 14. Variation of reflection energy with frequency in the PS section. Wells SS1 and SS202 are gas-producing wells and SS201 is a dry hole. 


\section{Discussion and conclusions}

We have presented a case study of anisotropic parameter estimation using PS-wave reflections and evaluating their characteristics associated with the gas reservoirs in sandstone and volcanic formations. The multicomponent data are acquired using digital MEMS (microelectro-mechanic-system) sensors. Although high-quality shear-wave data are obtained, the single-sensor data are contaminated with strong ground roll. The PS converted-wave data also show profound non-hyperbolic effects due to both the asymmetric raypath and the presence of VTI, as well as a significant amount of shear-wave splitting over the volcanic formation. Special tools and careful processing are required to handle these complications.

A model-based noise reduction technique (Qian and Zhao, 2003) is used to eliminate the ground roll and the technique is very effective compared with other conventional techniques. For processing the converted-wave in the presence of VTI, we used the recently developed Cxtools (Dai, 2003; Li et al., 2007) to handle the non-hyperbolic effects and build the velocity models required for converted-wave imaging. We have also shown that the VTI effects and the effects of the asymmetric raypath can be fully compensated for using the four-parameter theory of Li and Yuan (2003), which leads to an improvement in both imaging quality and event correlation between PP and PS converted-waves.

To evaluate converted shear-wave splitting for 2D or 3D data with a narrow-azimuth distribution, we have adopted a rotation-scanning procedure that maximizes the separation of the fast and slow split shear-waves. We have also utilized the concept of time-delay spectra that allows the generation of time-delay section. It is interesting that the amount of splitting determined from the data can be correlated to the known gas reservoirs.

In terms of other gas reservoir response, the PP-reflection is consistently weak and the PS-reflection is consistently strong in the presence of gas. Furthermore, low-frequency shadows can also be observed in the PP-section whilst they are absent in the PS-section. These are all consistent with the observation of shear-wave splitting correlated with gas accumulations, demonstrating the benefit of multicomponent seismic data from digital MEMS sensors.

\section{Acknowledgements}

We thank PetroChina Daqing Oilfield Ltd. for permission to show the data. We thank Qian Zhongping, Jinghua Zhang, Wang Guishui, Min Chunhua and for processing the data. This work is supported by the CNPC international collaboration programme and PetroChina Daqing 
through the Edinburgh Anisotropy Project (EAP) of the British Geological Survey (BGS), and is published with the approval of all project partners and the Executive Director of BGS (NERC).

\section{References}

Alford, R., 1986. Shear data in the presence of azimuthal anisotropy: Dilley, Texas: 56th Annual International Meeting, SEG, Expanded Abstracts , 476-479.

Angerer, E., Crampin, S., Li, X.Y. and Davis, T.L., 2002. Processing, modelling and predicting time-lapse effects of overpressured fluid-injection in a fractured reservoir Geophysical Journal International, 149, 267-280.

Calvert, A.S., Novak, J.M., Maher, J., Burch, D.N., Bird, D., Larson, R., 2005, A tale of two surveys: experiences processing two similar but different land 3D-3C MEMS surveys, $75^{\text {th }}$ SEG meeting, Houston, USA, Expanded Abstracts, 975-978.

Castagna, J. P., Sun, S. and Siegfried, R. W., 2003. Instantaneous spectral analysis: Detection of low-frequency shadows associated with hydrocarbons: The Leading Edge, 22, no. 2, 120-127.

Chapman, M. 2003. Frequency dependent anisotropy due to meso-scale fractures in the presence of equant porosity, Geophysical Prospecting, 51, 369-379

Chen, S. Li, X.-Y., Sun, X., Dai, H. and Jiang, P., 2007. PP and PS response from Volcanic gas reservoirs. First Break, 25, 35-41.

Crampin, S. and Lovel, J.H., 1991. A decade of shear-wave splitting in the Earth's crust: what does it means? what use can we make of it? and what should we do next? Geophysical Journal International 107, 387-407.

Dai H. 2003. Integrative analysis of anisotropy parameter and velocities for PS converted waves. 73rd SEG meeting, Dallas, USA, Expanded Abstracts, 1577-1580.

Gaiser, J. and Van Dok, R., 2001. Green River basin 3D/3C case study for fracture characterization: Analysis of PS-wave birefringence, 71st Ann. Internat. Mtg: Soc. of Expl. Geophys., 764-767.

Helbig, K. and Thomsen, L., 2005, 75-plus years of anisotropy in exploration and reservoir seismics: A historical review of concepts and methods: Geophysics, 70, 9ND-23ND.

Jiang, C., Chen, S., Zhang, E. and Zhong, D., 2004. Methodology and application of seismic prediction of gas-bearing volcanic reservoir: 74th SEG Meeting, Denver, Expanded Abstracts, 1595-1598.

Li, X.-Y., 1997. Fractural reservior delineating using multicomponent seismic data. 
Geophysical Prospecting, 54, 39-64

Li, X.-Y., 1998. Processing PP and PS waves in multicomponent sea-floor data for azimuthal anisotropy: theory and overview, Procedding of the Eighth International Workshop on seismic Anisotropy (Revue De L'institut francia du petrole), 53, 607-620

Li, X.-Y. and Crampin, S., 1993. Linear transform techniques for analysing split shear-waves in four-component seismic data. Geophysics, 58, 240-256

Li, X.-Y., Dai, H. and Mancini, F., 2007. Converted-wave imaging in anisotropic media: theory and case studies. Geophysical Prospecting, 55, in press.

Li, X.-Y. and Yuan, J. 2003. Converted-wave moveout and conversion-point equations in layered VTI media: theory and application. Journal of Applied Geophysics, 54, 297318.

Odebeatu, E., Zhang, J., Chapman, M., Liu, E. and Li, X. Y., 2006. Application of spectral decomposition to detection of dispersion anomalies associated with gas saturation: The Leading Edge, 25, no. 2, 206-210.

Qian, Z. and Zhao, B., 2003. A regular noise elimination method for prestack 3D seismic data: 73rd SEG Meeting, Expanded Abstracts, 2040-2043.

Vetri, L., Loinger, E., Gaiser, J., Grandi, A. and Lynn, H., 2003. 3D/4C Emilio: Azimuth processing and anisotropy analysis in a fractured carbonate reservoir. The Leading Edge, 22, 675-679.

Yuan, J., 2001. Analysis of four-component sea-floor seismic data for seismic anisotropy. $\mathrm{PhD}$ Thesis, University of Edinburgh. 